\title{
Arica und seine Verbindung mit dem bolivianischen Altiplano
}

Albert Leemann

\section{Die Eisenbahnverbindung Arica-La Paz}

Am 13. Mai 1967 konnte eine der kühnsten Gebirgsbahnen der Welt, die Bahn von Arica nach La Paz, den 54. Geburtstag ihrer Inbetriebnahme feiern. Der Schienenstrang führt von der nordchilenischen Hafenstadt Arica vorerst $10 \mathrm{~km}$ der Küste entlang und tritt dann ins Lluta-Tal ein, dessen üppig bepflanzte Talsohle sich als Flußoase scharf von den sie umgebenden, völlig vegetationslosen Wüstenhügeln abhebt. Bei km 70 beginnt der Steilanstieg in die Cordillera Occidental; auf einer Strecke von $42,7 \mathrm{~km}$ werden $2247 \mathrm{~m}$ Höhenunterschied mit Zahnrad überwunden. Das Trasse führt durch tiefe Felseinschnitte und zahlreiche Tunnel. Bei Puquios $(\mathrm{km} \mathrm{112})$ ist der Altiplano in einer Höhe von $3728 \mathrm{~m}$ erreicht. Einsame Indianerinnen hüten ihre Lamaund Alpakaherden auf der immensen Höhensteppe, der Puna. Schmelzwasserbäche von den schneebedeckten Fünftausendern mäandrieren durch die Ebene, die während der Regenzeit mit einem Anflug von Grün überzogen ist. Zu Füßen des TacoraVulkanes, bei der Station General Lagos (km 184,5), ist mit $4256 \mathrm{~m}$ der höchste Punkt erreicht. Höhenungewohnte Reisende leiden unter Kopfschmerzen und Atemnot; der mitgeführte Sauerstoff wird rege beansprucht. Zwischen den Stationen Visviri und Charaña passiert der Zug die chilenisch-bolivianische Grenze. $130 \mathrm{~km}$ östlich davon überquert der Schienenstrang den Rio Desaguadero, den Abfluß des Titicacasees. Eine nur 7,5 km lange, aber bedeutsame Seitenlinie zweigt in der Ortschaft General Pando (km 338; $3925 \mathrm{~m}$ ) nach Corocoro ab, dem Zentrum der hochgelegenen Kupferminen. Wichtigster Eisenbahnknotenpunkt der ganzen Strecke ist Viacha (km 415), wo die Linien Antofagasta-Guaqui und Arica-La Paz sich kreuzen. Der Blick auf die gigantischen Sechstausender der vergletscherten Cordillera Real wird frei (Illimani, Sorata, HuaynaPotosí, Mururata). Nach einem fühlbaren Anstieg über eintönige, vegetationsarme Schotterfelder steht man bei El Alto ( $\mathrm{km} \mathrm{438;4083} \mathrm{m)} \mathrm{plötzlich} \mathrm{völlig}$ überrascht vor dem $400 \mathrm{~m}$ bis $700 \mathrm{~m}$ tiefen, scharfrandig in die Ebene eingeschnittenen Talkessel. Aus der Vogelschau wird eine überwältigende Sicht auf die bolivianische Hauptstadt frei. Das Häusermeer von $\mathrm{La} \mathrm{Paz} \mathrm{nimmt} \mathrm{den} \mathrm{ganzen} \mathrm{Talgrund} \mathrm{und} \mathrm{die}$ niedrigen Terrassen in Anspruch. Auf dem geschützten, fruchtbaren Talboden gedeihen die er- sten Bäume. Im Osten wird die Szenerie durch den Illimani $(6457 \mathrm{~m})$ beherrscht. Der durch Erosion und Denudation in zahlreiche Einzelformen aufgelöste mineralreiche Kesselhang leuchtet in intensiven roten, gelben, grauen und blauen Farben. In vielen Kehren windet sich die Bergbahn von der Hochebene zur Endstation La Paz (km 440; $3701 \mathrm{~m}$ ) hinab.

"Mit dem Ziel, die politischen und kommerziellen Beziehungen beider Republiken auszudehnen, kommen Bolivien und Chile überein, den Hafen Arica und El Alto de la Paz mit einer Eisenbahnlinie zu verbinden. Die Konstruktion übernimmt innerhalb eines Jahres von der Ratifizierung dieses Vertrages an die chilenische Regierung auf ihre Kosten.» Dies ist der Wortlaut des Artikels III des Vetrages von 1904, durch den Bolivien von Chile die Konzession einer Bahnlinie zwischen Arica und La Paz zugesprochen wurde. Mit der 1913 eingeweihten Bahn erhielt das seit dem unglücklichen Salpeterkrieg (1879-1884) zum Binnenland gewordene Bolivien einen doppelten Ausgang an die chilenische Küste, hatte es doch schon vorher auf die Linie OruroAntofagasta zählen können.

Die neue Gebirgsbahn mit einem schwierigen Trasse von $440 \mathrm{~km}$ Länge und einer Steigung von $6,5 \% \mathrm{im}$ Zahnradabschnitt ist der kürzeste und eleganteste Zugang vom Pazifik nach $\mathrm{La} \mathrm{Paz}$, das einerseits mit dem Tiefland von Santa Cruz verbunden ist, einer Zone, die dank ihrer Erdöllagerstätten und der Produktion tropischer Agrarerzeugnisse berufen ist, eine bedeutende Rolle in der bolivianischen Wirtschaft zu spielen. Anderseits verschafft diese Bahnlinie dem Hafen an der chilenischen Küste einen neuen Aufschwung.

\section{Die bewegte Entwicklung der nordchilenischen Stadt Arica}

Aricas Entwicklung ist wechselvoll; es existierte bereits vor Ankunft der Spanier. Das Fischerdorf war durch einen Aimara-Stamm gegründet worden, erhielt aber später Zuzug von Quechua- und Atacameña-Indianern. Francisco Pizzarro sprach einen Teil dieses Territoriums dem ersten spanischen Erforscher Pedro Pizzarro als Encomienda zu. Bis gegen 1600 blieb Arica ein kleines Dorf. Dies änderte sich, als ein Beschluß des Vizekönigs verlangte, daß 


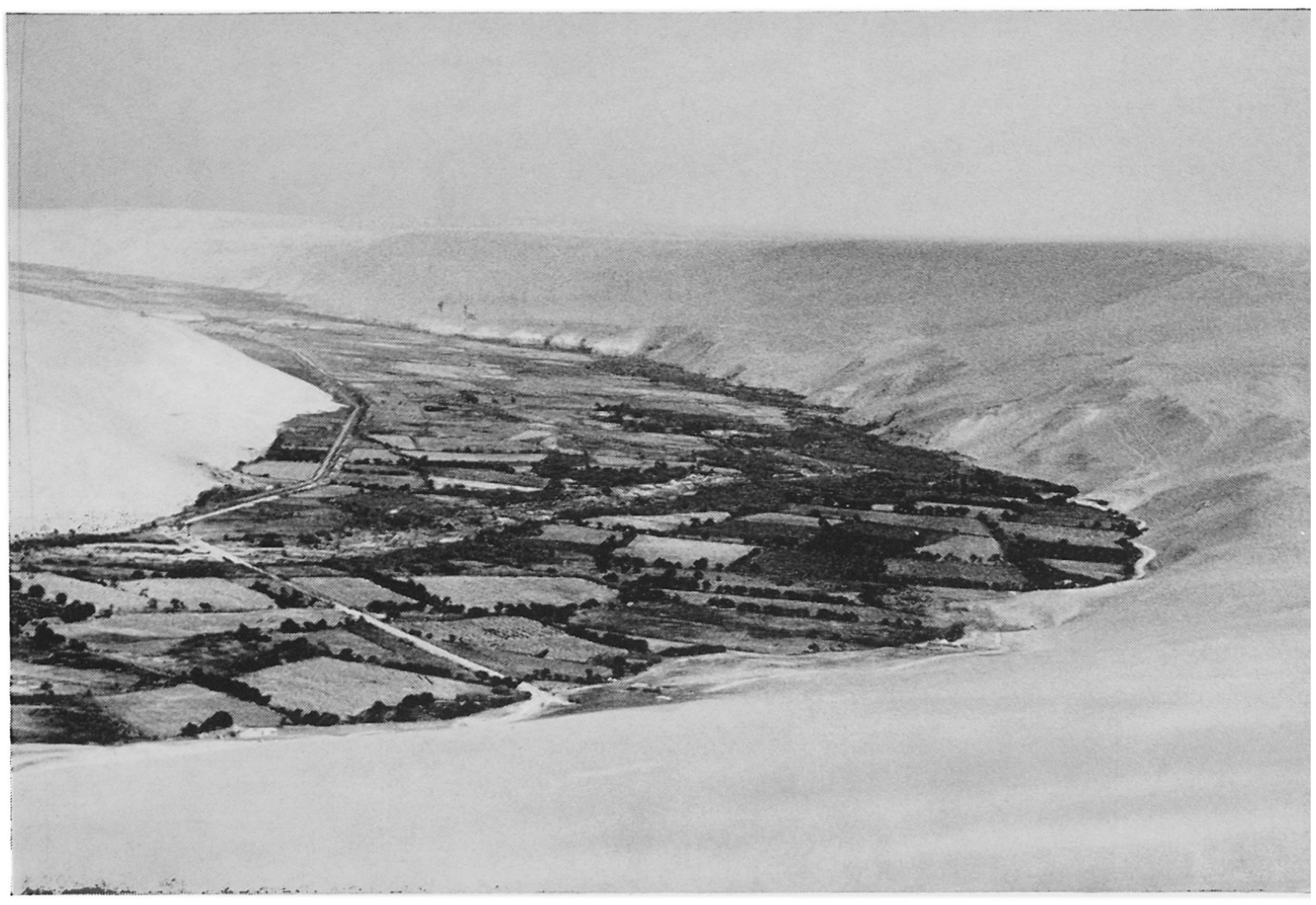

Abbildung 1. Das Lluta-Tal zieht sich nördlich Arica als Flußoase durch die nordchilenische Wüste

alle Quecksilberschiffe Arica anlaufen sollten. Dadurch wurde der Landweg nach den Erzvorkommen von Potosí um mehr als $80 \mathrm{~km}$ verkürzt. Die Siedlung nahm einen unerwarteten Aufschwung: sie wurde zum Transithafen für den gesamten Handel mit Alto Perú und erreichte eine Bevölkerung von 6000 Personen.

Die Erschöpfung der Silbererze von Potosí führte zum Zerfall des Handels über Arica. Um die Situation einigermaßen zu retten, dekretierte der König von Spanien durch eine Cédula Real den Puerto Franco de Arica.

Als Peru seine Unabhängigkeit erlangte, verleibte es Arica seinem Territorium als integrierenden Bestandteil ein. Bedeutsam ist der Umstand, daß damit der Handel mit Alto Perú (= Bolivien) unterbunden wurde. Bolivien suchte und fand seinen Ausgang zum Meer über den Hafen von Cobija. Arica verlor dadurch sein Hinterland und verwandelte sich in der Folge in ein unbedeutendes Dorf von nurmehr 3000 Einwohnern.

Der Ausbruch des Salpeterkrieges (1879) traf Arica in diesem Stadium des Abstiegs. Nach Beendigung des fünf Jahre währenden Streitfalles machte die Siegermacht Chile große Gebietsansprüche gegenüber den beiden Verlierern Peru und Bolivien gel- tend. So ging Bolivien seines ganzen wertvollen Atácama-Anteiles verlustig und wurde zum Binnenstaat. Im Vertrag von Ancón wurde festgehalten, daß das ehemals peruanische Tacna und Arica für zehn Jahre unter die Herrschaft Chiles fallen sollten. Nach Ablauf dieser Zeit sollte ein Plebiszit über die endgültige Staatszugehörigkeit entscheiden. Dieses fand nie statt, so daß der leidige Tacna-AricaStreitfall erst 1929 unter den beteiligten Staaten direkt geregelt werden konnte. Die Bestimmungen halten fest, daß Tacna unter die peruanische, Arica endgültig unter die chilenische Herrschaft fallen solle.

Unter der chilenischen Verwaltung gewann Arica seine frühere Stellung wieder langsam zurück. Um die Wirtschaft zu beleben, erklärte die Regierung durch ein präsidiales Dekret Arica 1954 zum Freihafen. In der Folge zogen Tausende von Siedlern nach dem chilenischen Norden, um hier eine neue Existenz aufzubauen. Heute wird auf den meisten Einfuhrartikeln wieder Zoll erhoben, obwohl Wirtschaftskreise dringend eine echte Zona Franca fordern. Gegenwärtig zählt Arica ungefähr 70000 Einwohner. Ein legaler Organismus, die Junta de Adelanto, hat es sich zur Aufgabe gemacht, die Stadt zu entwickeln. Die definitive Stabilisierung soll 


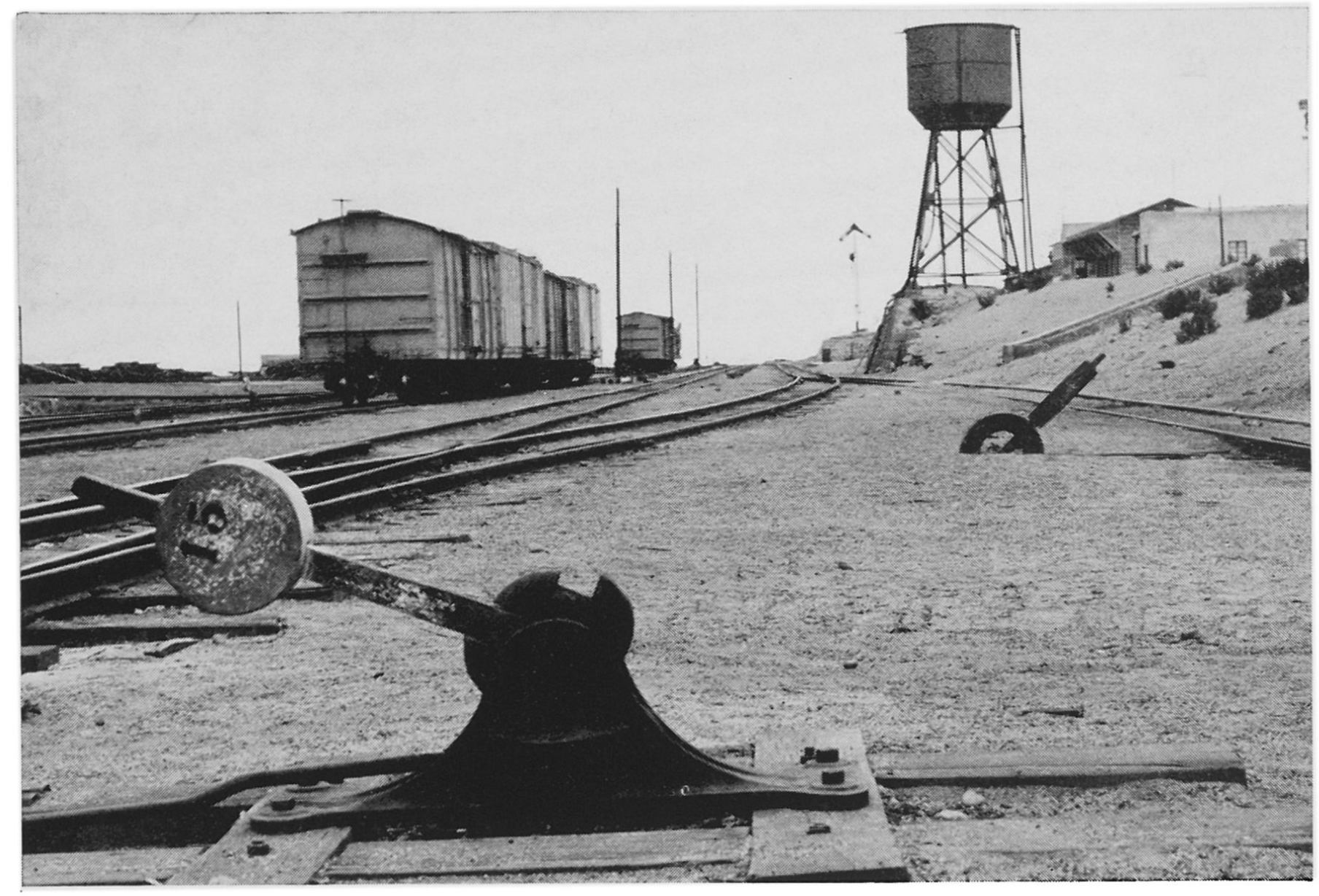

Abbildung 2. Hohe Wassertürme, einfache Unterkünfte für das Personal, davor ein kleines Blumenbeet, weite Abstellgeleise charakterisieren die Bahnstationen des Altiplano. Im Zahnradabschnitt müssen die ursprünglichen Zugskonvois in kleine Kompositionen aufgeteilt werden; Equipenwechsel werden vorgenommen. Station Puquios (km 112,5; 3728 m)

durch das Estatuto de Arica erreicht werden, das vorsieht, die Stadt zu einem der wichtigsten Häfen der Pazifikküste auszubauen.

\section{Der A usbau des veralteten Hafens von Arica}

Der Hafenausbau ist dringend notwendig. Die zwei 1949 konstruierten Pfahlmolen erlaubten den Frachtern kein direktes Anlegen am Quai. Die Fracht mußte durch mühsame Umladeoperationen und mit unzulänglichen Mitteln gelöscht werden. Die Jahreskapazität des Hafens betrug daher 1965 lediglich $180000 \mathrm{t}$. Welches sind die Unzulänglichkeiten in den Hafenoperationen, die nicht nur von den bolivianischen Importeuren, sondern auch von den chilenischen Kaufleuten beanstandet worden sind? Das umständliche Löschen spielte sich folgendermaßen ab:

1. Der Frachter ankert einige $100 \mathrm{~m}$ vor der Mole. Die Fracht wird vom Meerschiff in Leichter umgeladen (= Lanchaje-System).

2. Der Leichter fährt zur Mole; Löschen der Ladung.
3. Transport von der Mole zum Lagerplatz; Umladen.

4. Transport vom Lagerplatz ins Lagergebäude; Ausladen.

Um den Vorgang noch weiter zu komplizieren, wurde selbst die Fracht, die an eine einzige Firma konsigniert war, durch verschiedene Umschlagfirmen gelöscht. Das flüchtige und unordentliche Entladen im Zusammenhang mit den vielen Zwischenstationen führte nicht nur zu übermäßigen Warenverlusten, sondern auch zu bedeutenden Verzögerungen in der Ablieferung, was ständig Reklamationen seitens der Verbraucher zur Folge hatte. Da der Hafen nicht mit einer genügenden Anzahl Waagen ausgerüstet war, die für das unmittelbare Wägen aller konsignierten Waren eingesetzt werden konnten, war es unmöglich, die Verluste, die sich normalerweise an Bord ergeben, beweiskräftig zu erfassen.

Ein anderer Faktor, der die Einfuhr Boliviens über Arica erschwert, sind die enorm hohen Unkosten im Hafen. Es existiert keine vernünftige Preisbasis, geschweige denn ein fester Hafentarif, auf den sich die Importeure verlassen könnten. Die Gebühren 


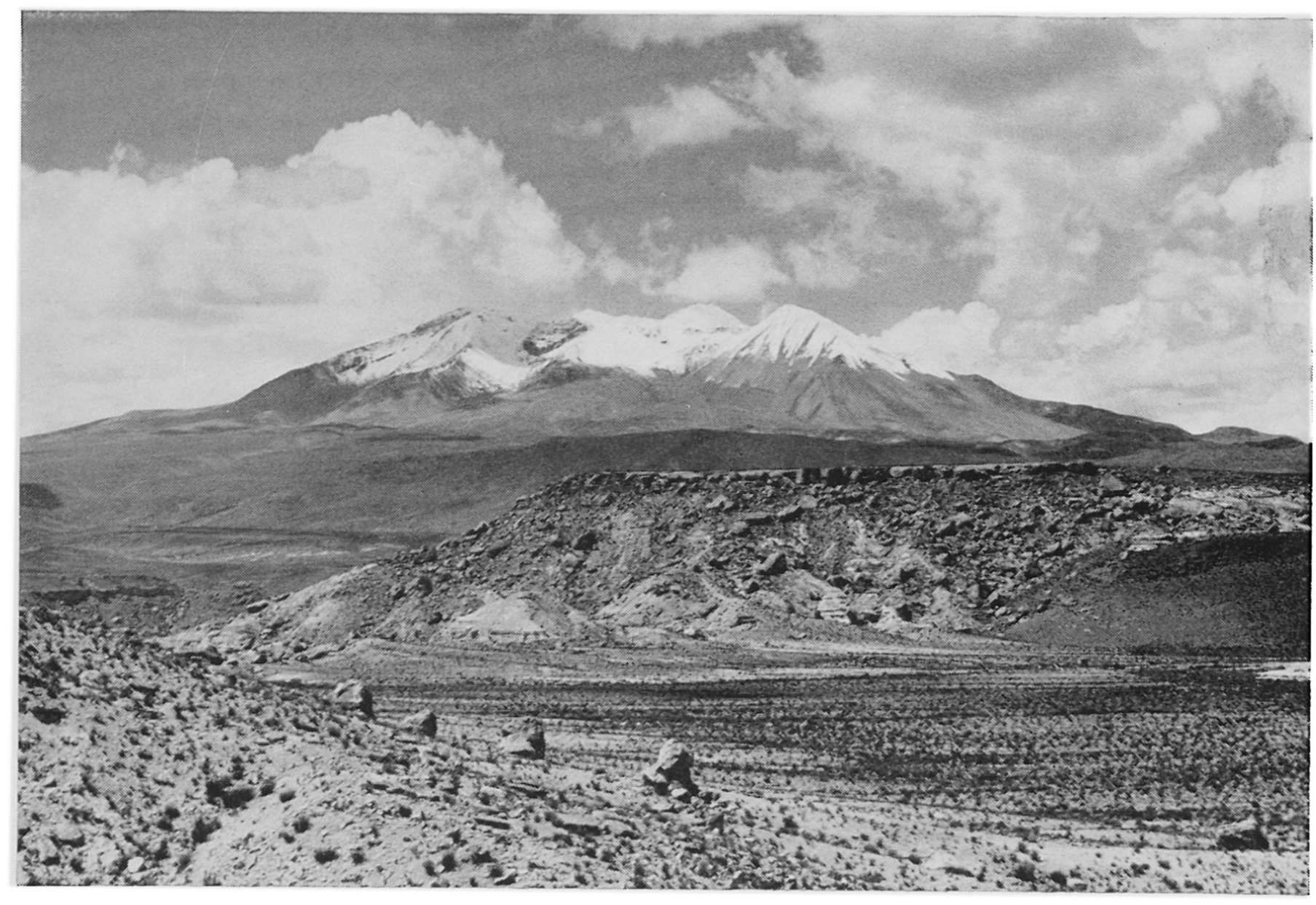

Abbildung 3. Schmelzwasserbäche von den schneebedeckten Fünftausendern mäandrieren über den Altiplano und schneiden Terrassen aus den Schotterfluren

wechseln willkürlich von einem Verladen zum andern, obwohl es sich um die gleichen Artikel handelt. Die unberechenbaren Verladekosten bewirken eine bedeutende Verteuerung des Produktes am Bestimmungsort. Dadurch wird die Konkurrenzfähigkeit des Hafens stark eingeschränkt.

Die bolivianische Kritik macht aber auch nicht vor den landeseigenen Institutionen halt. So wird zum Beispiel der Agencia Aduanera del Consulado de Bolivia im Hafen von Arica vorgeworfen, ihre Aufsichtspflicht nur mangelhaft zu erfüllen. Um einen wirkungsvolleren Arbeitsablauf zu garantieren, schlägt man eine Reorganisation dieses Amtes vor. Zugleich soll der Personalbestand durch kompetente Kräfte ergänzt werden.

Nach einer Ubereinkunft zwischen der Junta de Adelanto de Arica (J. A. A.) und dem Ministerio de Obras Públicas wurde 1960 mit dem Ausbau des Puerto Comercial de Arica begonnen. Hauptziel dieses fundamentalen Werkes ist es, das direkte Anlegen von Frachtern mittlerer und größerer Tonnage $\mathrm{zu}$ ermöglichen, somit das antiquitierte und mühsame Leichter-System aufzuheben. Seit dem Juni 1965 ist die erste Phase der Hafenerneuerung in Betrieb, die das direkte Anlaufen eines tiefgängigen Frachters erlaubt; 1966 ist das gleichzeitige Löschen von zwei größeren und einem kleineren Frachtschiff möglich geworden. Der vollständige Ausbau sieht das gleichzeitige Anlegen von fünf tiefgängigen Schiffen vor, 10 bis $12 \mathrm{~m}$ Wasserstand bei Ebbe sollen auch großen Erzfrachtern das direkte Anlegen am neuen, $1000 \mathrm{~m}$ langen Quai erlauben. Die 30 ha dem Meer abgewonnenen Landes gestatten ein leichteres und wirtschaftlicheres Lagern und Umladen der Waren. 1966 ist mit dem Bau der Lagergebäude begonnen worden. Das Hafenareal wird mit einem festen Belag überzogen. Zusätzliche Gebäulichkeiten für Personal, Polizei, Zoll- und Hafenbehörden werden erstellt. Die Modernisierung der Hafenausrüstungen sieht den Einsatz von sechs elektrischen Kränen vor; 30 große Schleppeinheiten sollen im Lagerareal Verwendung finden. Ohne gröBere Mechanisierung erlauben die neuen Hafeneinrichtungen einen Umschlag von annähernd 1 Mio t im Jahr.

Damit der enorme Aufwand des Hauptausbaues ermessen werden kann, seien die bis anfangs 1966 verwendeten Materialien aufgeführt: $5000 \mathrm{t}$ Stahl, 40000 t Portland-Zement, 3 Mio t Steinblöcke; 4,5 Mio t Erdbewegungen. Der Maschinenpark hat einen Wert von 4,8 Mio US-Dollar. 600 Arbeiter aus Chile, Bolivien und Peru arbeiteten während 


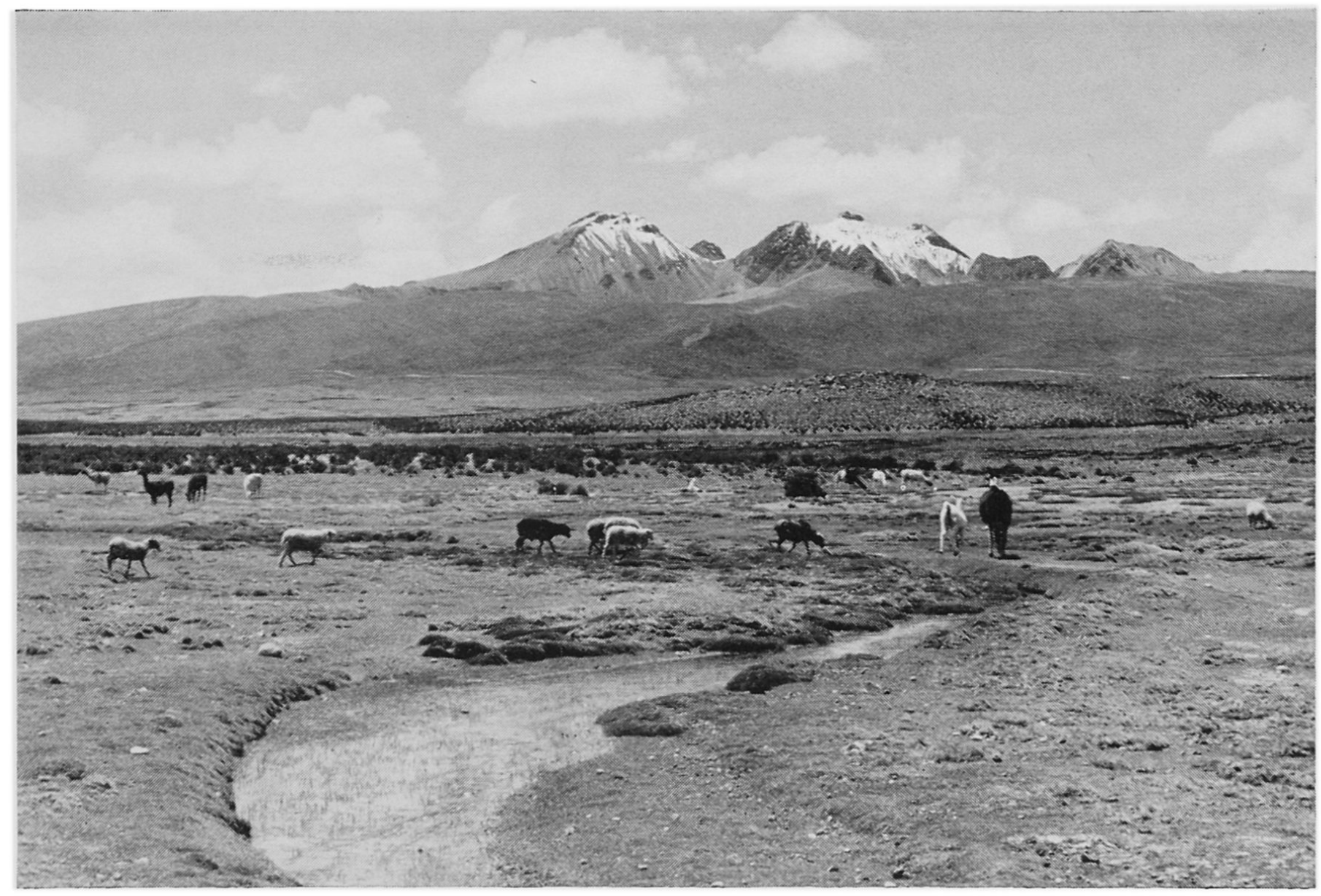

Abbildung 4. Einsame Indianer hüten ihre Lama-, Schaf- und Alpakaherden auf der immensen Höhensteppe, die während der Regenzeit mit einem Anflug von Grün überzogen ist. Zwischen Humalpaca $(\mathrm{km} \mathrm{160,4;} 4083 \mathrm{~m})$ und Villa Industrial $(\mathrm{km} \mathrm{176,0;4231} \mathrm{m)}$

sechs Jahren im Schichtbetrieb. Die Kosten für die Basisarbeiten des Hafens von Arica werden auf ungefähr 20 Mio US-Dollar geschätzt. Für Ergänzungsarbeiten sowie Installationen und Hafenausrüstungen sollen weitere 20 Mio US-Dollar investiert werden. In dieser Summe ist die Konstruktion einer automatischen Schüttgut-Verladevorrichtung inbegriffen, die den Export von Erzen mittleren und niedrigen Gehaltes erlauben würde; diese Mineralien mußten bisher der hohen Frachtkosten wegen vom internationalen Markt ferngehalten werden. Die Umschlagsmenge könnte durch die Verwendung entsprechender Einrichtungen für das Getreideausladen zusätzlich erhöht werden. Studienpläne sehen eine weitere Möglichkeit in der Errichtung eines Lagers für Erdöl und Erdölderivate, die sich durch Pipelines befördern lassen.

Die Hafenrenovation äußert sich nicht nur in einer vollständigen Neuausstattung mit modernen Werkgeräten und der Einstellung kompetenten Personals, sondern auch in der Tatsache, daß sich die Hafenbehörde veranlaßt sieht, die Arbeitsmethoden zu vereinfachen und den Arbeitsablauf sowohl in den Verladeoperationen als auch in der amtlichen Ausfertigung der Warendokumente zu rationalisieren. Die chilenischen Behörden beraten die Ausarbeitung eines Tarifsystems, durch das sich das Total der Hafen- und Eisenbahnkosten genau bestimmen läßt. Von dieser Situation bis zum Import cif $\mathrm{La} \mathrm{Paz}$ wäre nur ein kleiner Schritt zu tun. Damit würde ein schon lange gehegter Wunsch des bolivianischen Importhandels erfüllt. Im Bemühen um größte Erleichterung im Transitverkehr vom Hafen Arica aus schlägt die bolivianische Handelskammer die Schaffung einer Zollstation in $\mathrm{La} \mathrm{Paz}$ vor. Dies bedingt die Errichtung spezieller Lagergebäude für bolivianische Transitprodukte im Hafenareal.

Als stimulierendes Mittel zur Entwicklung Aricas wird die Schaffung einer Zollfreizone gewünscht, wie sie das Proyecto de Estatuto vorschlägt. Die Studie der Freizone ist vor allem für den Gang der bolivianischen Wirtschaft bemerkenswert: einerseits wären Waren und Nahrungsmittel zu Preisen des Ursprungslandes - oder noch billiger - erhältlich; anderseits könnten bolivianische Produkte in einer Industriefreizone zum Export verarbeitet werden und damit auch der Wirtschaft Aricas einen neuen Impuls verleihen. Als aussichtsreich erscheint die Projektierung einer Anlage zur Anreicherung minderwertiger Erze. 


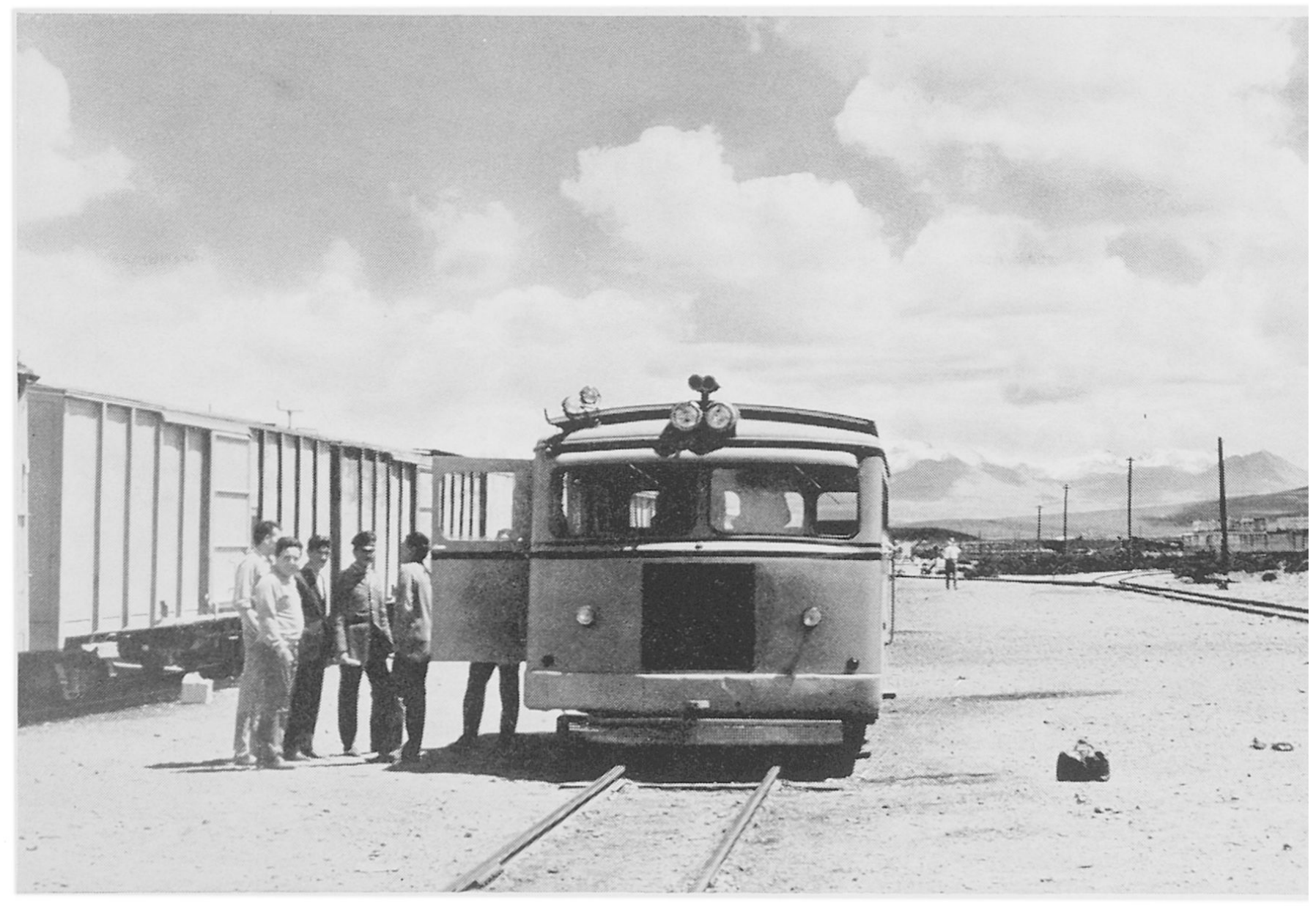

Abbildung 5. Wer Glück hat, kann die Fahrt von Arica nach La Paz in weniger als zehn Stunden in diesem kleinen Schienenbus zurücklegen. Neun Reisende und der Chauffeur finden darin Platz; individuelle Foto. halte auf der Strecke sind gestattet

\section{Die Rückständigkeit der Eisenbahn; Pläne zu ihrer Modernisierung}

Die Hafenkapazität hat sich gegenüber früher bedeutend gesteigert. Obwohl die Eisenbahn ihre Traktion von Dampf auf Dieselelektrizität umstellte, hat ihre Leistung mit dieser Entwicklung nicht Schritt halten können, was zur Folge hat, daß in Arica beständig ungefähr $3000 \mathrm{t}$ Waren mit Destination La Paz lagern. Die logische Reaktion der Importfirmen auf den Verzug in der Frachtverteilung ist eine Abweichung von diesem Weg; die Transportmenge der Bahn nahm in letzter Zeit ab. Für viele Waren wurden Umwege über andere Häfen in Kauf genommen. Die behördlichen Vorschläge, die Straßenverbindung Tacna- La Paz auszubauen, dürften die Bahnunternehmung nicht gleichgültig lassen.

Die Bahn ist 1913 im Einklang mit den Erfordernissen der Bauepoche ausgerüstet worden. 1943 beschloß die chilenische Regierung, die Bahn, die bis dahin von einer autonomen Administration verwaltet worden war, der Empresa de los Ferrocarriles del Estado einzuverleiben. Seit dieser Zeit ist der Wunsch wach, das Transportsystem durch eine voll- ständige Modernisierung den heutigen technischen Erfordernissen anzupassen. In der gleichen Richtung wirkt auch die bolivianische Empresa Nacional de Ferrocarriles, die nach der Verstaatlichung der Bahnen auch für den Abschnitt Charaña-La $\mathrm{Paz}$ verantwortlich ist. Nebst bestimmten Sofortmaß. nahmen soll ein erweitertes Programm verwirklich1 werden, das in der Planung zeitlich mit der vollen Kapazitätsausnützung des Hafens von Arica zusammenfällt. Als erstes ist das Traktionsvermöger durch die Anschaffung zweier zusätzlicher diesel. elektrischer Lokomotiven erhöht worden. Die boli. vianischen Staatsbahnen planen die Anschaffun neuer Autorails. Andere Maßnahmen zielen daraul hin, das eingesetzte Rollmaterial zu verbessern. Sc wurden zum Beispiel 200 neue Wagenachsen zuı Verfügung gestellt. Der Ausbau der Reparaturwerk. stätten von Chinchorro erlaubt einen raschen unc fachgerechten Unterhalt des Bahnmaterials. Die un. gefähren Totalkosten der chilenischen Sofortmaß. nahmen machen annähernd eine Million US-Dol. lar aus.

Um uns ein Bild von der Vergrößerung des Trans. portvolumens zu machen, müssen wir uns die Um. schlagskapazität des neuen Hafens in Erinnerung 


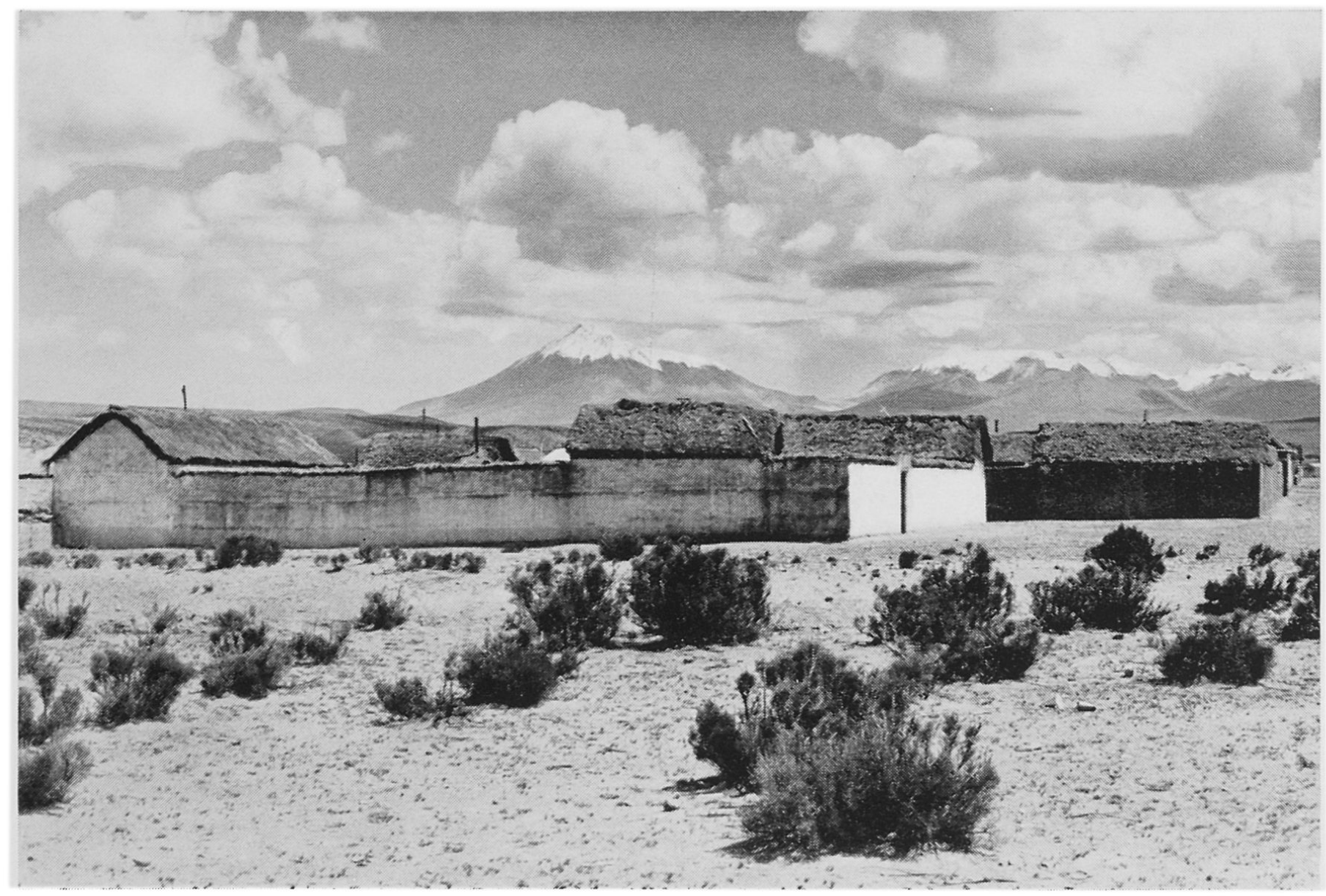

Abbildung 6. Kleine Indianerdörfer verlieren sich in der Puna. Im Hintergrund nahe der chilenisch-bolivianischen Grenze erkennt man einige Fünftausender, darunter den steilen Kegel der Tacora

rufen: sie wird auf rund 1 Mio t im Jahr geschätzt. Reduzieren wir diese Größe vorsichtshalber um $20 \%$ bis $30 \%$ und zählen wir zudem den internen Bedarf des Departementes von Arica ab, der sich auf ungefähr $150000 \mathrm{t}$ im Jahr beläuft, so läßt sich die zukünftige Jahreskapazität der Eisenbahn mit $500000 \mathrm{t}$ bis $600000 \mathrm{t}$ im Jahr veranschlagen. Dies würde einer Steigerung um das Sieben- bis Achtfache gegenüber der Frachtmenge der letzten Jahre gleichkommen. Es erscheint als vernünftig, daß die Pläne zur Anpassung an die neuen Bedürfnisse auf einer Größenordnung fußen, die der wachsenden Entwicklung auf mindestens 20 Jahre hinaus gerecht zu werden vermag. Ein entsprechende Vergrößerung des Frachttransportes könnte mit den Traktionsmitteln, die bisher in den Zahnradabschnitten zum Einsatz kamen, bei weitem nicht bewältigt werden. Das von den Experten des Programmes Chile -California in Angriff genommene Programm diskutiert vier Möglichkeiten:

Im ersten Vorschlag wird die Traktion mit Adhäsionslokomotiven für die Normalstrecken und speziellen Diesellokomotiven für die Zahnradabschnitte besprochen. Diese Lösung ist die limitierteste in bezug auf die Leistungssteigerung, erfordert aber auch die geringste Totalinvestition (4,5 Mio USDollar).

Der zweite Vorschlag sieht die Verwendung dieselelektrischer Lokomotiven im Zahnradabschnitt vor. Der gesteigerten Leistung stehen erhöhte Kosten gegenüber; die Verbesserung der Geleiseanlagen inbegriffen, rechnet der Voranschlag mit einem Aufwand von 6,5 Mio US-Dollar. Als Nachteil bei der Verwirklichung von Projekt 1 und 2 wird die mangelnde Erfahrung im Bau solcher spezieller Lokomotiven herausgehoben.

Als dritte Lösung wird die Elektrifizierung aller Zahnradabschnitte und damit der Einsatz elektrischer Zahnradlokomotiven erwogen. Das ausgebaute Hydroelektrizitätswerk von Chapiquiña kann die geforderte Leistung abgeben. Die Kosten dieses Projektes, inbegriffen die Verbesserung der Geleiseanlagen, belaufen sich auf ungefähr 8 Mio USDollar. Es ist von Vorteil, daß man sich von vorneherein ein genaues Bild über die Wirtschaftlichkeit dieses zu verwendenden Lokomotivtyps machen kann.

Die vierte Lösung endlich könnte die Limitierung der Schleppkapazität voraussichtlich ganz beheben. Sie sieht die Neuanlage des Trasses zwi- 


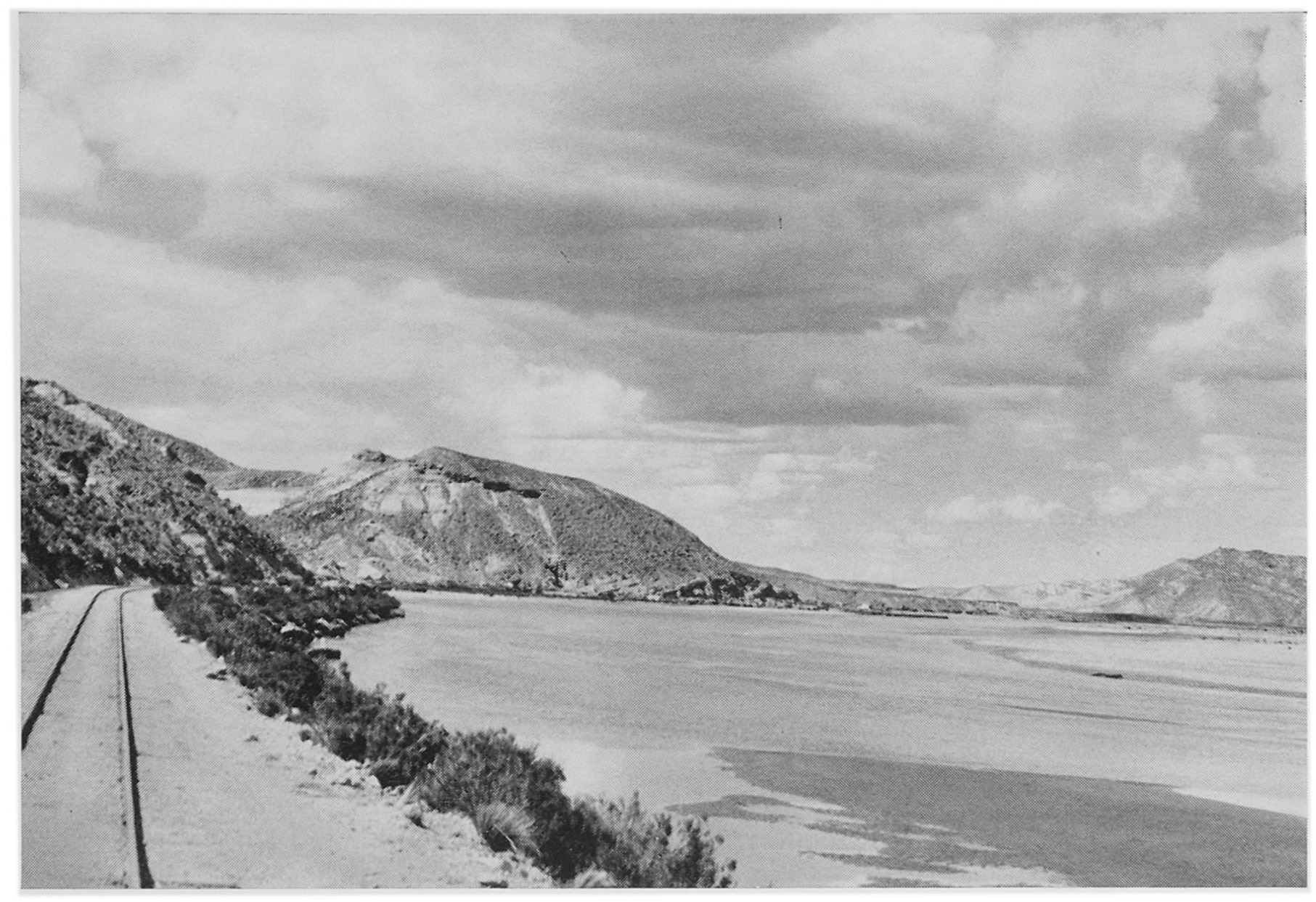

Abbildung 7. Auf der Fahrt durch den bolivianischen Altiplano überquert der Schienenstrang den Desaguadero, den Abfluß des Titicacasees

schen den Stationen Central (bisher km 69,8; $1418 \mathrm{~m}$ ) und Puquios (bisher km 112,5; $3728 \mathrm{~m}$ ) vor. Die Steigung dürfte maximal $3 \%$ betragen, so daß die gesamte Traktion mittels normaler Adhäsionslokomotiven erfolgen könnte. Dieser Vorschlag ist bezüglich der Steigerung der Frachtkapazität zweifellos der wirkungsvollste, erfordert aber alles in allem einen Aufwand von über 14 Mio USDollar.

\section{Die enormen Kosten des Entwicklungsprogrammes}

Wenn wir diesen Kosten die Investitionen beifügen, die der Hafenausbau erfordert, ergibt sich für das chilenische Entwicklungsprogramm des HafenBahn-Komplexes eine Totalsumme von ungefähr 50 Mio US-Dollar. Im Estatuto de Arica wird die Initiative zur Fusionierung der Eisenbahn AricaLa Paz (chilenischer Sektor) und des Hafens von Arica gutgeheißen. Man erhofft davon nicht nur ein abgestimmtes Funktionieren von Schiffsverlad und Bahntransport, sondern auch eine einheitliche rationelle Finanzierung durch Aufnahme gemeinsamer Anleihen. Da diese Investitionen die Mittel eines Landes übersteigen, ist Chile auf die finan- zielle Beteiligung des Auslandes angewiesen. Die ersten Kontakte mit der Weltbank und der BID (Banco Interamericano de Desarrollo) scheinen die Realisierung der weitgesteckten Ziele positiv zu beeinflussen.

\section{Die Verbesserung des Verbindungsnetzes}

Die Erhöhung der Transportkapazität läßt ebenfalls den Wunsch nach besseren Kontaktmöglichkeiten sowohl zwischen den beiden Bahnabschnitten als auch zwischen den Handelspartnern aufkommen. Erst 1961 begann die chilenische CORFO (Corporacione de Fomento de la Produccion) mit dem Aufbau eines primären nationalen Netzes für große Distanzen zwischen den Städten, die der Verbindungen am dringendsten bedurften. Seit 1962 funktionieren die Verbindungen zwischen Santiago und den Städten Arica im Norden und Coyhaique im Süden. 1963 beschloß die chilenische Regierung die Beschleunigung des Stammnetzausbaues, was Ende 1964 zur Schaffung der ENTEL-CHILE (Empreso Nacional de Telecomunicaciones S.A.) führte. Ein Kurzwellennetz zwischen Santiago und Arica, Iquique, Antofagasta, Coyhaique und Puerto Mott wird ausge- 


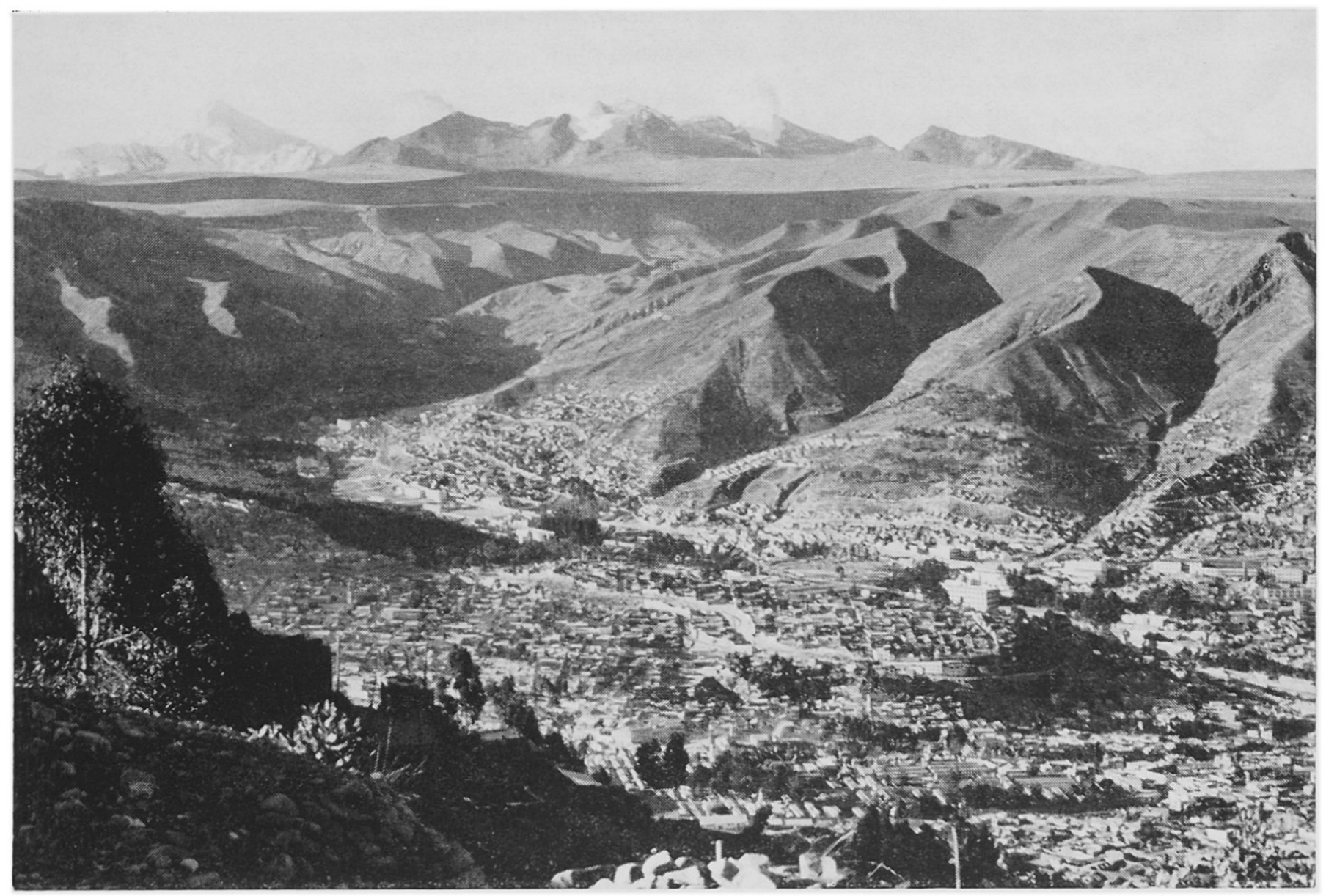

Abbildung 8. Ausblick von der Schotterebene bei El Alto ( $\mathrm{km} 438 ; 4083 \mathrm{~m}$ ) auf den scharfrandig eingeschnittenen Talkessel von La Paz. Im Hintergrund die vergletscherten Sechstausender der Cordillera Real

baut. 22 Telefon- und 12 Telexkanäle sollen die Verbindung sowohl für den zivilen als auch den militärischen Gebrauch zwischen den Städten Arica und Iquique gewährleisten. Zwischen der im Dezember 1965 geschaffenen ENTEL-BOLIVIA und der ENTEL-CHILE bestehen Kontakte, die der Beschleunigung desVerbindungsausbaus zwischen Santiago-La Paz und Arica-La Paz dienen. Ein von der Comisión de Arica und Cámara de Industria y Comercio de Bolivia ratifiziertes Übereinkommen betrifft die Einrichtung eines privaten Telefonnetzes, durch das eine größere Flexibilität im gesteigerten Handelsaustausch erreicht werden soll. Zudem wird eine Verbesserung der Flug- und Postverbindungen angeregt, die bis heute als ungenügend empfunden werden.

\section{Die Aufgaben der Junta de Adelanto de Arica (J. A. A.)}

Die erwähnten bedeutenden Entwicklungsarbeiten im chilenischen Norden erfordern eine wirkungsvolle Planung und Koordination der verschiedenen Arbeitsprogramme. Aus diesem Grunde ist die Junta de Adelanto de Arica (J. A. A.), als juristische
Person und mit Eigenkapital versehen, geschaffen worden. Ausgaben und Investitionen der J.A.A. figurieren im chilenischen Jahresvoranschlag in globaler Form; die detaillierte Aufteilung ist Angelegenheit der Institution. Die J. A. A. reiht sich in die dezentralisierten Organismen ein, die im Artikel 107 der Constitución Política Chilena vorgesehen sind. Sie ist ganz allgemein beauftragt, die Entwicklung des Departementes von Arica durch die Vorbereitung und Verwirklichung von Werken zu fördern, die sie für das Wohlergehen der ländlichen und städtischen Bevölkerung als wesentlich erachtet. Nebst der Entwicklung von Handel und Industrie soll auch der Prospektion von Bodenschätzen die notwendige Beachtung geschenkt werden.

Die Arbeit der Junta kann in folgende Punkte gegliedert werden:
Abteilung Konstruktion:
1. Puerto Comercial de Arica
2. Hydroelektrizitätswerk von Chapiquiña
3. Bau von Wohnungen

Abteilung Öffentlichkeit:

1. Finanzierung des Planes zur Erneuerung von Trinkwassernetz und Kanalisation 
2. Programm zur Einführung der Elektrizität in den Dörfern

3. Förderung des Erziehungswesens durch Bau von Schulen, Erwerb von Schulmaterial, Unterstützung der Universitäten del Norte und de Chile

\section{Spezielle Projekte:}

Darlehen an Bergbauunternehmungen und chemische Industrien. Unterstützung von Industrieprojekten, Hotelbauten, Transportunternehmungen. Als Beispiel der Investition im sozialen Wohnungsbau seien die Verbesserungen oder der Neuaufbau provisorischer Wohnviertel («marginales») erwähnt. Eine erste Etappe dieses Programmes sieht den Bau sanitärer Einheiten und der Außenmauern vor; die restlichen Arbeiten sollen zur Hauptsache durch die Besitzer selbst ausgeführt werden, die materiell und technisch unterstützt werden durch die J. A. A. Kredite wurden unter anderem auch für folgende Zwecke gewährt: Erweiterung des Straßennetzes, Flußverbauungen, Ausbau des Flugplatzes, Agrarreformen. Investitionen und Ausgaben der Junta werden durch Steuereinnahmen ermöglicht, die durch die Gesetze 13.039 und 14.824 geregelt sind. Die Steuergelder stammen von Importabgaben aus dem Hafen von Arica, von Gebühren auf Produkten, die aus Rohstoffen oder aus Teilen ausländischer Herkunft in Arica hergestellt worden sind und nach dem übrigen Chile verkauft werden, sowie aus Abgaben beim Kauf und Verkauf beweglicher Güter. Zudem kommen der Junta 50\% des Gewinnes aus dem Casino von Arica zu (die restlichen 50\% werden unter den Universitäten de Chile und del Norte aufgeteilt!). Die J. A. A. hat ferner Anspruch auf $4 \%$ der Wettgelder aus dem Hippodrom von Arica.

Die Bestimmungen des Estatuto de Arica führen eine Reihe von Mitteln auf, die der Gegend einen ausschlaggebenden Impuls verleihen sollen. Bekanntlich genießt das Departement gewisse Freiheiten, um in seiner Zone Industrien anzusiedeln. Diese Bestimmungen krankten bisher an der mangelnden Stabilität, was die Investition des nötigen Unternehmerkapitals in einer modernen Manufakturindustrie verhindert hat. Das Estatuto de Arica versucht, dieses Unvermögen zu korrigieren; sein Hauptvorschlag bezweckt, jegliche Investition, die zur Industrieentwicklung beiträgt, zu ermuntern. Zudem sind Dispositionen zur Förderung des Handels mit Bolivien getroffen worden. Alle diese vielseitigen Initiativen zielen dahin, Arica sowohl zu einem bedeutenden Regionalzentrum als auch zu einem wichtigen südamerikanischen Pazifikhafen auszubauen.

\section{Literaturverzeichnis}

1. Ferrocarril Arica-La Paz 1966 (Programas de la oficina de Relaciones Públicas del Ferrocarril Arica-La Paz, Sección Chilena).

2. Harms Erdkunde, Band V, Amerika (1966).

3. The South American Handbook 1967. 